\title{
Decision-Making Deficits of Korsakoff Patients in a New Gambling Task With Explicit Rules: Associations With Executive Functions
}

\author{
Matthias Brand, Esther Fujiwara, and \\ Sabine Borsutzky \\ University of Bielefeld
}

\author{
Elke Kalbe and Josef Kessler \\ Max-Planck-Institute for Neurological Research
}

\author{
Hans J. Markowitsch \\ University of Bielefeld
}

\begin{abstract}
Decision-making deficits reflected by risky decisions in gambling tasks have been associated with frontal lobe dysfunctions in various neurologic and psychiatric populations. The question remains whether decision-making impairments are related to executive functions. The authors developed a new gambling task, the Game of Dice Task, with explicit and stable rules for reinforcement and punishment, to investigate relations between executive functions and risk-taking behavior in an explicit decision-making situation. A sample of 35 alcoholic Korsakoff patients and 35 healthy controls was examined with the Game of Dice Task and a neuropsychological test battery. Results show that Korsakoff patients are strongly impaired in this explicit decision-making task and that these disturbances are correlated with specific executive functions.
\end{abstract}

Keywords: decision making, risk-taking behavior, categorization, cognitive flexibility, feedback processing, Korsakoff syndrome

Patients with frontal lobe damage as well as patients with frontal dysfunctions (due to substance dependence, for example) show deficits in decision making and an insensitivity to future consequences in gambling tasks (Bechara et al., 2001). However, the potential impact of specific executive functions on performance in gambling tasks is still under debate (Bechara \& Martin, 2004).

The Iowa Gambling Task, developed by Bechara, Damasio, Damasio, and Anderson (1994), is one of the most frequently used gambling tasks. With this test or modified versions, various frontal lobe-damaged populations have been shown to exhibit decisionmaking deficits characterized by a high tendency for risky decisions. Impairments have been described in patients with ventromedial prefrontal cortex injuries (Bechara et al., 1994; Bechara, Tranel, \& Damasio, 2000; Manes et al., 2002; Sanfey, Hastie, Colvin, \& Grafman, 2003); those with frontal dysfunctions caused by alcohol or cocaine addiction (Adinoff et al., 2003; Bechara \&

Matthias Brand, Esther Fujiwara, Sabine Borsutzky, and Hans J. Markowitsch, Department of Physiological Psychology, University of Bielefeld, Bielefeld, Germany; Elke Kalbe and Josef Kessler, Max-PlanckInstitute for Neurological Research, Cologne, Germany.

Additional materials are on the Web at http://dx.doi.org/10.1037/08944105.19.3.267.supp

We thank Martina Konrad (Haus Grefrath), Sabine Kühn (Haus Eller), Andrea Schwerdt (Therapiezentrum Germersheim), Markus Mendt (TPR Köln), and Hans-Peter Steingass (Haus Remscheid) for arranging the examination of the Korsakoff patients. Furthermore, we are very grateful for Alexandra Hovaguimian's corrections of the English. We also thank Antoine Bechara for his very helpful comments.

Correspondence concerning this article should be addressed to Matthias Brand, Department of Physiological Psychology, University of Bielefeld, P.O. Box 100131, 33501, Bielefeld, Germany. E-mail: m.brand@unibielefeld.de
Damasio, 2002; Bechara et al., 2001; Bechara, Dolan, \& Hindes, 2002; Bolla et al., 2003); and individuals with Huntington's disease (Stout, Rodawalt, \& Siemers, 2001), obsessive-compulsive disorder (Cavedini, Riboldi, D'Annucci, et al., 2002), pathological gambling (Cavedini, Riboldi, Keller, D'Annucci, \& Bellodi, 2002), and psychopathy (Mitchell, Colledge, Leonard, \& Blair, 2002).

In the Iowa Gambling Task, possible choices (selection of cards from different decks) are either advantageous or disadvantageous, but each choice is full of ambiguity. Prior to selection, it is impossible to exactly calculate the outcome (amount of gain or loss) of each choice. The rules for gains and losses are implicit; thus, participants have to learn to avoid the disadvantageous choices and prefer the advantageous by using the feedback (the amount of gain or loss) following each trial (for detailed description of the task and its theoretical background, see Bechara, 2003). Accordingly, dysfunctions in this gambling task have been interpreted as impairments in using feedback from previous trials for current decisions. However, it is still unclear whether executive functions, such as categorization of different alternatives, influence decision-making processes, at least in a decision-making situation with more explicit rules for gains and losses.

Considering real-life situations, decisions followed by reinforcement or punishment often depend on explicit probabilities, and therefore, strategic procedures and specific executive functions maintain substantial impact. For example, when people drive in a car on a street with a speed limit, they know that if speeding is being monitored the punishment is higher the faster they drive. They also know they should slow down when they see a police car to avoid punishment. In such situations, executive functions such as planning, categorization, and set-shifting will influence the decision-making process and outcome to a major degree. Though executive functions are not defined as a consistent entity (Miyake 
et al., 2000), Smith and Jonides (1999) have suggested five main components of executive functions: attention and inhibition, task management, planning, monitoring, and coding. Furthermore, working memory involving the temporary storage and manipulation of new information may also be a part of executive functions. At least it influences subcomponents of executive functions to a major degree (see D'Esposito \& Grossman, 1996).

We suggest that in a gambling task with explicit and stable rules, the aforementioned subcomponents of executive functions play an important role. For instance, to detect advantageous and disadvantageous alternatives, different obvious winning probabilities of choices and their associated amounts of money must be categorized and compared. Therefore, attention to the relevant stimuli and inhibition of irrelevant stimuli is necessary. Furthermore, participants may plan to win as much money as possible and have to decide which choices will lead to goal attainment. Probably, task performance also involves monitoring of current behavior and, if necessary, strategy changing and switching to other choices associated with higher gains or lower losses long term. However, feedback processing and use of previous feedback for current decisions are also important in a gambling task with explicit and stable rules, as both can trigger a long-term strategy change. The latter aspect is similar to the feedback value in the Iowa Gambling Task.

The aim of the present study was to analyze the possible influence of such executive functions on decision making in a gambling situation with explicit and stable rules for gains and losses, as well as for winning probabilities. For this purpose, we studied alcoholic Korsakoff patients, a group in which former substance dependence led to brain damage, probably including frontal lobe dysfunctions and, accordingly, neuropsychological impairments. In research into the causation of alcoholic Korsakoff syndrome (KS), it is discussed whether direct neurotoxic effects of ethanol or thiamine deficiency or a combination is the main contributing factor (Lishman, 1990). Though alcohol abuse is the most frequently reported etiology of KS, it can also be caused by other diseases, such as anorexia (Becker, Furman, Panisset, \& Smith, 1990), or by gastrectomy (Shimomura, Mori, Hirono, Imamura, \& Yamashita, 1998). Furthermore, the extension of structural and functional brain changes and their corresponding neuropsychological deficits is still under debate. Classically, neuropathological changes in patients with KS comprise diencephalic degenerations, such as in the mammillary bodies and medial thalamic nuclei. In addition, the cerebellum and periaqueductal and periventricular gray matter are affected (Cravioto, Korein, \& Silberman, 1961; Mair, Warrington, \& Weiskrantz, 1979; Malamud \& Skillicorn, 1956; Victor, Adams, \& Collins, 1989). More recently, frontal lobe damage and hippocampal volume reductions have also been reported in alcoholism and KS (Kril, Halliday, Svoboda, \& Cartwright, 1997; Moselhy, Georgiou, \& Kahn, 2001; Sullivan \& Marsh, 2003). Accordingly, KS patients exhibit a wide range of neuropsychological impairments in addition to the well-described memory deficits. Although severe and persistent anterograde memory impairment and deterioration of the sense of time constitute the cardinal symptoms of KS (World Health Organization, 1994), executive functions often associated with the frontal lobes can also be affected (Brand, Kalbe, Fujiwara, Huber, \& Markowitsch, 2003; Joyce \& Robbins, 1991; Kopelman, 1991; Krabbendam et al., 2000). Furthermore, dysfunctions in affective processing have been observed (Brand, Fujiwara, et al., 2003; Markowitsch, Kessler, Bast-Kessler, \& Riess, 1984; Markowitsch, Kessler, \& Denzler, 1986).

We hypothesized that KS patients would show disturbances in a newly developed gambling task. In this task, rules for gains, losses, and winning probabilities are explicit and stable during the entire task duration, affording the application of long-term strategy to increase the outcome. Patients with KS were conjectured to make more risky decisions than were healthy controls because of their frontal lobe dysfunctions. Specifically, we hypothesized that KS patients' performance in the gambling task with explicit rules would be correlated with the specific executive functions of categorization, set-shifting, and cognitive flexibility, as well as with anterograde memory. Generation and application of a long-term strategy, which is important in decisions made under uncertainty about the outcome, could be affected by both disturbances in executive functions and anterograde memory deficits.

\section{Method}

\section{Statistical Analyses}

All statistical analyses were carried out with the Statistical Package for the Social Sciences (SPSS) version 10.0 for Windows (Release 10.0.7; Chicago, SPSS Inc.). In case of normally distributed data, we used parametric methods ( $t$ tests and Pearson correlations), and in case of significant deviations from the normal distribution (tested with the KolmogorovSmirnov test), we used corresponding nonparametric methods (chi-square test, Mann-Whitney $U$ tests, and Spearman correlations). To adjust for multiple comparisons, we corrected the results (Bonferroni). For analyzing the frequency of single choices in the gambling task, we conducted a multivariate analysis with repeated measurements (multivariate analysis of variance) with choice as a within-subject factor and group as a betweensubjects factor.

\section{Participants}

A total of 35 patients with clinically diagnosed alcoholic KS according to ICD-10 (World Health Organization, 1994) and DSM-IV (American Psychiatric Association, 1994) criteria (for alcohol-induced amnesic syndrome or alcohol-induced persisting amnesic disorder, respectively) and 35 healthy controls (CG) without neurologic or psychiatric history were enrolled in the study. The CG contained staff members of the cafeteria and the administration of the University of Bielefeld, as well as their relatives and friends. The KS patients were recruited from different homes for chronically multi-impaired addicts of the Allgemeine Hospitalgesellschaft in Germany (in the cities Cologne, Duesseldorf, Grefrath, and Germersheim). Neither the control participants nor the KS patients received financial compensation for their participation. All patients underwent an extensive neurologic and psychiatric examination, carried out by the phy-

\section{Table 1}

Age, Sex, and Education of the Korsakoff Syndrome (KS)

Patients and the Control Group (CG)

\begin{tabular}{lccc}
\hline \multicolumn{1}{c}{ Variable } & $\mathrm{KS}(n=35)$ & $\mathrm{CG}(n=35)$ & $p$ \\
\hline Mean $(S D)$ age in years & $56.7(6.5)$ & $56.8(6.1)$ & .970 \\
Sex & & & \\
$\quad$ Male & 26 & 25 & .788 \\
$\quad$ Female & 9 & 10 & \\
Mean $(S D)$ education in years & $9.5(0.9)$ & $10.7(1.2)$ & $<.05$ \\
\hline
\end{tabular}


sicians of the different cooperating institutions. Any illicit drug consumption was a criterion for exclusion for the controls. Regular alcohol consumption (fewer than six standard drinks per week) and regular caffeine or nicotine consumption were allowed for the controls. Twenty-one of the control participants were regular smokers. The history of alcohol consumption of the KS patients was revealed by checking medical documentations and by interviewing their relatives and caregivers. All KS patients had extensive alcohol consumption over continuous periods of more than 14 years. They did not, however, exhibit typical signs of dementia and were therefore not diagnosed as patients with alcohol-related dementia according to the criteria of Oslin, Atkinson, Smith, and Hendrie (1998). Twentynine of the KS patients were tobacco smokers, and with the exception of 1 female participant, all patients consumed caffeine regularly. For both the patient group and the controls, participants with further (current or preceding) neurologic or psychiatric symptoms were excluded from the study. The groups were matched regarding age and sex. The controls had slightly higher education than the KS group. However, there was no significant effect of education on performance in the experimental task (see the
Results section). In addition, there was no effect of nicotine consumption on task performance in either group. Mean age, sex, and education of both groups are shown in Table 1. In the KS group, there was 1 left-handed subject (male), and in the control group, all participants were right-handed.

\section{Neuropsychological Test Battery}

An extensive neuropsychological test battery was administered to the patients (see Table 2). The general cognitive state was determined by a German version (Kessler, Markowitsch, \& Denzler, 1990) of the MiniMental State Examination (Folstein, Folstein, \& McHugh, 1975) and the Clinical Dementia Rating Scale (Hughes, Berg, Danziger, Coben, \& Martin, 1982).

Components of executive functions were assessed with the Wisconsin Card Sorting Test-Modified Version (mWCST; Nelson, 1976), which is assumed to measure categorization, set-shifting, and tendency to perseverate, as well as cognitive flexibility and the ability to use feedback (see Spreen \& Strauss, 1998). Interference susceptibility was examined with the

Table 2

Results of the Neuropsychological Test Battery

\begin{tabular}{|c|c|c|c|}
\hline Measure & $\operatorname{Max}$ & KS & CG \\
\hline \multicolumn{4}{|l|}{ General cognitive state } \\
\hline Mini-Mental State Examination & 30 & $25.5(2.5)$ & $\geq 27$ \\
\hline Clinical Dementia Rating Scale & 3 & $0.5(0-0.5)$ & $0(0-0)$ \\
\hline \multicolumn{4}{|l|}{ Memory } \\
\hline \multicolumn{4}{|l|}{ Memo } \\
\hline Immediate recall & 10 & $5.3(1.0)$ & $7.29(0.73)$ \\
\hline Delayed recall & 10 & $1.0(0-6)$ & $6.67(1.45)$ \\
\hline \multicolumn{4}{|l|}{ Digit Span } \\
\hline Forward & 9 & $5.6(0.8)$ & $\geq 5$ \\
\hline Reversed & 9 & $4.0(0.7)$ & $\geq 4$ \\
\hline Rey-Osterrieth Figure delayed recall & 36 & 4.9 (4.6) & 16.6 \\
\hline \multicolumn{4}{|l|}{ Visuoconstructive abilities } \\
\hline Rey-Osterrieth Figure copy & 36 & $29.7(5.1)$ & 33.1 \\
\hline \multicolumn{4}{|l|}{$\begin{array}{l}\text { Executive functions and information } \\
\text { processing }\end{array}$} \\
\hline \multicolumn{4}{|l|}{ Modified Wisconsin Card Sorting Test } \\
\hline Correct & 48 & $28.6(6.7)$ & \\
\hline Errors & & $13.7(6.3)$ & $12-13(t=41)^{\mathrm{a}}$ \\
\hline Perseverations & & $5.7(2.3)$ & $8-9(t=42)^{\mathrm{a}}$ \\
\hline \multicolumn{4}{|l|}{ Word Color Interference Test ${ }^{\mathrm{b}}$} \\
\hline Words & & $16.5(3.5)$ & $22-23($ per $11-22)$ \\
\hline Colors & & $25.5(7.7)$ & $36-41$ (per $11-22)$ \\
\hline Interference & & 54.7 (21.9) & $74-90($ per $11-22)$ \\
\hline Interference - colors & & $29.3(17.8)$ & $43-59($ per $11-22)$ \\
\hline \multicolumn{4}{|l|}{ FAS Test total } \\
\hline \multicolumn{4}{|l|}{ Intelligence } \\
\hline \multicolumn{4}{|l|}{ German adaptation of the National } \\
\hline Adult Reading Test ${ }^{\mathrm{c}}$ estimated IQ & & $104.9(13.1)$ & $109.6(12.8)$ \\
\hline German version of the Wechsler Adult & & & \\
\hline $\begin{array}{l}\text { Intelligence Scale }{ }^{\mathrm{d}} \text { Information } \\
\text { subtest }\end{array}$ & 24 & $13.6(4.3)$ & $17.0(5.2)$ \\
\hline
\end{tabular}

Note. Data are means (and standard deviations) except for Korsakoff syndrome (KS) group data for the clinical Dementia Rating Scale and Memo delayed recall, which are medians (and ranges), and control group (CG) data for Mini-Mental State, memory tests (except Memo) visuoconstructive abilities, and the Word Color Interference test, which are norm group scores. Clinically impaired functions (scores lower than cutoff scores or $t$ scores of 40, or percentile of 15 , or more than two standard deviations lower than control group or norm group) are indicated by boldface values. Max $=$ maximum; per $=$ percentile; FAS $=$ letters of the lexical verbal fluency task.

${ }^{a}$ Norm scores of Lineweaver et al. (1999). ${ }^{\mathrm{b}}$ German: Farb-Wort-Test. ${ }^{\mathrm{c}}$ German: Mehrfachwahl-WortschatzIntelligenztest. ${ }^{\mathrm{d}}$ German: Hamburg-Wechsler-Intelligenztest für Erwachsene. 
A

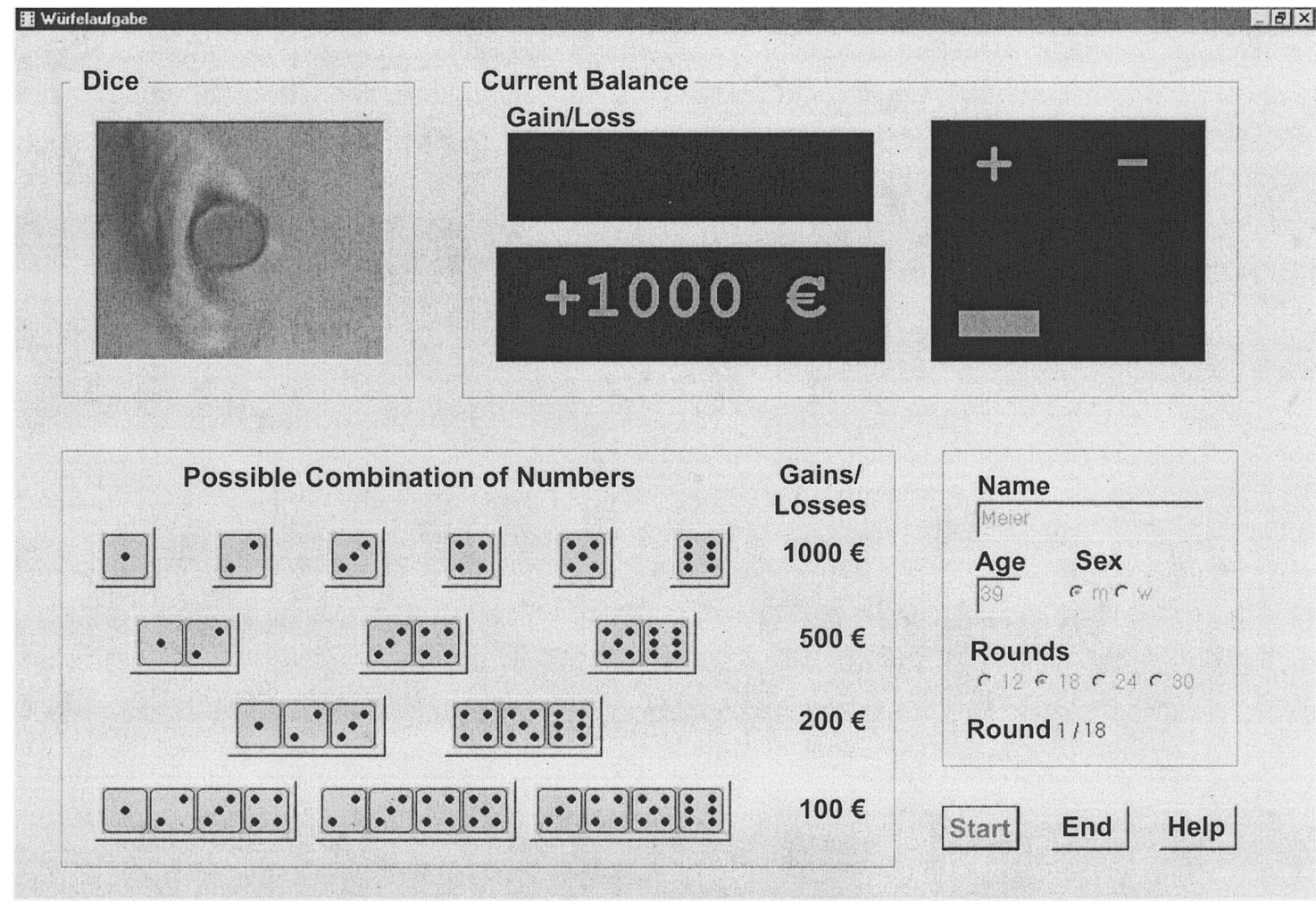

B

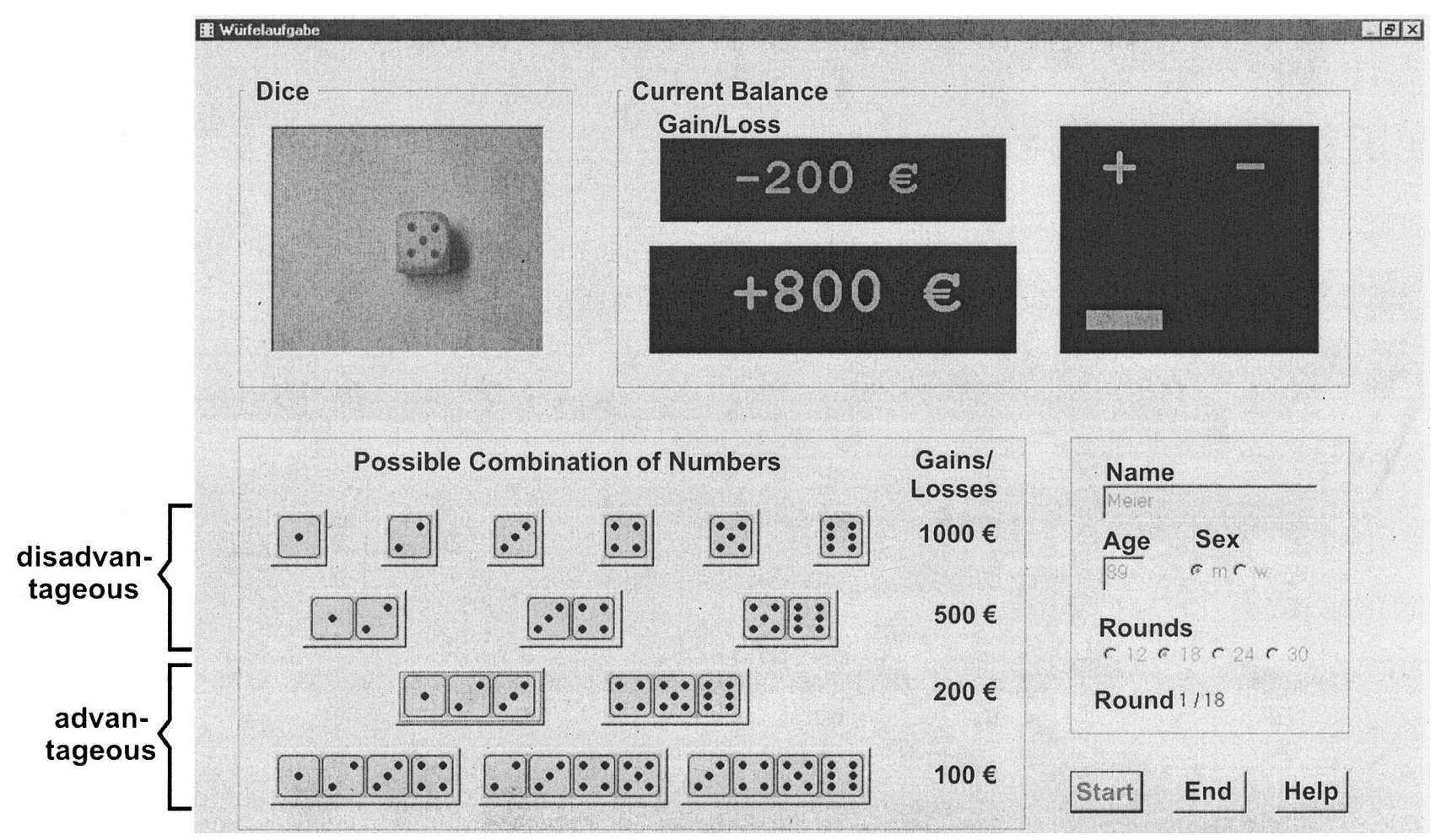


Interference Trial of the Word Color Interference Test (Farb-Wort-Test of the Nürnberger-Alters-Inventar; Oswald \& Fleischmann, 1997). The FAS Test (Spreen \& Strauss, 1998) was administered to assess verbal fluency, which is considered sensitive to executive dysfunctions (Bryan \& Luszcz, 2000). To study speed of information processing (speed of reading and color naming), we used the Word Trial and the Color Trial of the Word Color Interference Test (Oswald \& Fleischmann, 1997). Verbal short- and long-term memory were assessed with a verbal selective reminding task (Memo Test; Schaaf, Kessler, Grond, \& Fink, 1992). Additional standard tests were applied to assess verbal short-term and working memory (Digit Span forward and reverse of the German version of the Wechsler Adult Intelligence Scale-Revised [HAWIE-R]; Tewes, 1991), figural long-term memory (Rey-Osterrieth Figure [ROF] Test delayed recall; Osterrieth, 1944, according to Lezak, 1995), and general knowledge (Information subtest of the HAWIE-R; Tewes, 1991). Intelligence was estimated with a German adaptation of the National Adult Reading Test (Nelson, 1982), the Mehrfachwahl-Wortschatz-Intelligenztest (Lehrl, Merz, Burkhard, \& Fischer, 1991).

Control participants were examined with a reduced test battery including the Game of Dice Task, as well as several tests assessing different important neuropsychological functions (intelligence, verbal fluency, general knowledge). For administered tests, means and standard deviations of control participants are given in Table 2. Otherwise, scores of the norm groups were used.

\section{Decision Making in a New Gambling Task: The Game of Dice Task}

To assess risk-taking behavior, we constructed a new gambling task, the Game of Dice Task, based on the ideas of the aforementioned Iowa Gambling Task of Bechara et al. (1994) and the Risk Task of Rogers et al. (1999). The new task was designed to assess the possible influence of executive functions on decision making in a gambling situation. In the computerized Game of Dice Task, a virtual single die and a shaker are used. The subjects are asked to increase their fictive starting capital $(1,000$ $€[\sim \$ 1,307]$ ) within 18 throws of the die. Before each throw, subjects have to choose a single number or a combination of numbers (two, three, or four numbers). Each choice is related to specific fictive gains and losses that depend on the probability of occurrence of choice (a single number: 1,000 $€$ gain/loss; combination of two numbers: $500 €[\sim$ \$653] gain/loss; combination of three numbers: $200 €[\sim \$ 261]$ gain/loss; combination of four numbers: $100 €[\sim \$ 130]$ gain/loss). The rules and extent of gains and losses are explicitly described and visualized (see Figure 1A; a color version is available on the Web at http://dx.doi.org/10.1037/08944105.19.3.267.supp). The winning probability of the different choices can be reasoned easily by means of the ratio of occurrence (1:6, 2:6, 3:6, and 4:6). Therefore, the amount of risk associated with each choice is obvious (e.g., in the choice of a single number, there is a 1:6 chance to win 1,000 $€$ but a 5:6 chance to lose $1,000 €$, whereas in the choice of four numbers, there is a 4:6 chance to win $100 €$ but only a 2:6 chance to lose $100 €$ ). Participants are also informed that they have to make a total of 18 decisions. After each throw, the gain - in case of congruence between the selected number (or numbers) and the thrown number, or the loss-in case of incongruence between the selected number (or numbers) and the thrown number, and the altered capital are presented visually and pointed out by two different acoustic signals (gain $=$ jingle of a cash desk; loss $=$ dull noise). Furthermore, the number of remaining throws is also shown on the screen. The results of the throws are pseudorandomized, meaning that each of the six possible numbers occur three times during task performance, but in a balanced order. The maximum capital at the end of the game is 19,000 $€([\sim \$ 24,824]$ if the participant chooses a single number and is successful in each throw). The maximum deficit can be $-17,000 €([\sim \$ 22,213]$ if the participant chooses a single number and is unsuccessful in each throw).

To analyze risky decisions, we classified the choices of one or two numbers (probability of winning less than $50 \%$ and high gains but also high penalties) as risky or disadvantageous, respectively, whereas we classified the choices of three and four numbers (probability of winning $50 \%$ and higher, low gains but also low penalties) as nonrisky or advantageous (see Figure 1B; a color version is available on the Web at http://dx.doi.org/ 10.1037/0894-4105.19.3.267.supp). Besides this, the frequency of each chosen alternative (one number, two numbers, three numbers, or four numbers) can also be analyzed separately.

\section{Differences Between the Game of Dice Task and the Iowa Gambling Task as Well as Rogers' Risk Task}

The main difference between Bechara's Iowa Gambling Task (Bechara et al., 1994) and the Game of Dice Task concerns the explicitness of the rules for gains and losses in the latter. The different probabilities of the choices that win or lose money can be reasoned easily, and the different amounts of gains and losses according to their probability of occurrence are obvious. Accordingly, whereas the Iowa Gambling Task measures decisions made under ambiguous conditions (see Bechara, 2003), the Game of Dice Task assesses decisions made under uncertain or risky conditions. Furthermore, in contrast to the Iowa Gambling Task, in the Game of Dice Task participants are told that they have 18 throws to increase their starting capital, and the number of previous throws is shown on the screen. Similar to Bechara's task, in the Game of Dice Task some choices may lead to high short-term gains but will result in a long-term negative balance because of their low probability of reward. On the other hand, there are choices that lead to moderate short-term gains but to a positive balance long term because of their higher probability of reward.

In contrast to Rogers' Risk Task (Rogers et al., 1999), the gains and losses related to the different choices are stable and obvious for the participants during the entire duration of the Game of Dice Task. In Rogers' Risk Task, an array with a mixture of six red or blue colored boxes is presented on a computer screen, and subjects have to decide whether a yellow token is hidden in a red box or a blue box. The ratio of red and blue boxes as well as the balance of reward and punishment vary from trial to trial. However, in trials with an unequal ratio of red and blue boxes, the least likely outcome is always associated with the larger reward, but the amounts of gains and losses are not consistently associated with the probability of winning. Thus, participants have to react on the basis of presented probability and the related gain/loss in each trial but do not have

Figure 1 (opposite). The Game of Dice Task. A: The start position. Before each throw, participants have to choose a single number (e.g., 3) or a combination consisting of two numbers (e.g., 3 and 4), three numbers (e.g., 4, 5, and 6), or four numbers (e.g., 3, 4, 5, and 6). After each choice, the die is tossed (animated on the screen), and thereafter the result is shown (B). At the same time, an acoustic signal indicates whether the throw was successful, and the extent of gain or loss is shown (gains in green, losses in red). In addition, the altered capital is shown (also a positive balance in green and a negative balance in red). The choices of one single number and two numbers together are classified as disadvantageous (winning probability less than $50 \%$; high gains but also high losses), whereas the choices of three and four numbers together are classified as advantageous (winning probability $50 \%$ and higher; low gains but also low losses). 
the chance to deal with long-term strategies. However, they may apply short-term strategies to decide whether a provided probability together with a specific reward relation is advantageous or not. This is in contrast to the gain/loss relation according to winning probability in the Game of Dice Task. For example, in the Game of Dice Task, the most likely choice (four numbers together, winning probability of 4:6) is related to $100 €$ gain or loss in each of the 18 trials. Accordingly, the most risky alternative (one single number, winning probability 1:6) is associated with $1,000 €$ gain or loss in each trial. The participants can choose each of the 14 different alternatives (clustered in four groups with different probabilities and gains and losses) in each trial while the winning probabilities and amounts of gains and losses associated with each alternative remain stable during the entire task duration, as described above. Thus, the Game of Dice Task is more complex on a cognitive level and includes the possibility of planning decisions over the entire duration of the procedure and applying long-term strategies to maximize outcome.

\section{Results}

\section{Neuropsychological Test Battery}

Results in the neuropsychological test battery are shown in Table 2. The KS group exhibited severe verbal and figural anterograde memory deficits (shown in the delayed recall of the Memo Test and the delayed recall of the ROF). Though not clinically relevant, they also showed lower performances in verbal fluency (FAS Test) and general knowledge (Information subtest of the German adaptation of the Wechsler Adult Intelligence Scale) compared with controls. Mean performance in the mWCST was unimpaired according to the criteria of Lineweaver, Bondi, Thomas, and Salmon (1999).

\section{Risky Decisions in the Game of Dice Task}

The frequency of disadvantageous decisions in the controls deviated significantly from normal distribution, thus all statistical analyses including this variable were carried out with nonparametric tests. The KS group selected the disadvantageous choices (one single number or two numbers together, winning probability less than $50 \%$, high gains but also high losses) significantly more often than the controls $(\mathrm{KS}$ : median $=12.0$, range $=1-18$; CG: median $=2.0$, range $=0-18 ; U=245.0, p<.001$; see Figure 2). Considering the mode of the frequency of risky choices, the intergroup difference was even more obvious (KS: mode $=18$; CG: $\operatorname{mode}=1), \chi^{2}(16, N=70)=32.7, p=.008$. There was no effect of sex in either the KS group or the controls (KS: men's median $=11.0$, range $=2-18 ;$ women's median $=13.0$, range $=$ $1-18 ; U=117.0, p=.999 ;$ CG: men's median $=1.0$, range $=$ $0-18$; women's median $=8.0$, range $=0-16 ; U=78.0, p=$ .091). In the KS group, but not in the controls, the frequency of risky decisions was correlated with age (KS: $\rho=.371, p=.028$; CG: $\rho=.075, p=.688$ ). Correlations with years of education were not found in either group (KS: $\rho=-.271, p=.115$; CG: $\rho=$ $-.289, p=.093$ ). Furthermore, in neither the KS group nor the controls was there a significant difference in the Game of Dice Task performance between smokers and nonsmokers (all $p \mathrm{~s}>$ $.05)$.

As shown by the higher frequency of disadvantageous decisions in the KS group compared with the CG, the final outcome differed significantly between the two groups $(\mathrm{KS}:$ median $=-1,700 €$, range $=-12,500$ to $+1,900 € ; \mathrm{CG}$ : median $=+500 €$, range $=$ $-6,900$ to $+2,200 € ; U=138.5, p=.004)$.

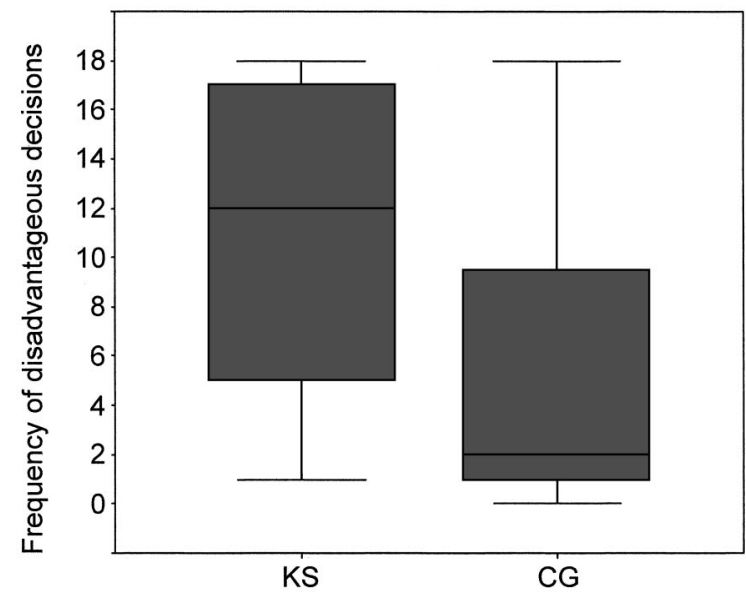

Figure 2. Median and range of frequency of disadvantageous decisions in the Korsakoff syndrome (KS) group and the control group (CG). Line across the box denotes the median, area in the box denotes the medial quartiles, and T-lines represent range of values.

Though the frequency of choices of each separate alternative (one single number, two numbers together, three numbers together, and four numbers together) in the Game of Dice Task was not normally distributed in the controls, we conducted an analysis of variance with repeated measurements, with choice as a withinsubject factor and group as a between-subjects factor. This parametric analysis was chosen because no comparable nonparametric method is available. For choice, there was no significant overall effect, $F(3,70)=1.71, p=.166$, but there was a significant Choice $\times$ Group interaction, $F(3,70)=9.84, p<.001$. Figure 3 demonstrates that patients more often selected the very risky choice (one single number) and the risky choice (two numbers together), whereas control participants selected the least risky choice (four numbers) to a higher degree. All single comparisons between both groups regarding frequency of selection of each single alternative were highly significant except for the alternative three-number choice (one number: $T=2.96, p=.004$; two numbers: $T=2.43, p=.017$; three numbers: $T=-0.75, p=$ .453 ; four numbers: $T=-5.01, p<.001)$.

\section{Correlations Between Decision Making and Neuropsychological Functions}

In the KS group, the frequency of disadvantageous decisions in the Game of Dice Task was highly correlated with the financial outcome $(\rho=-.78, p<.001)$. Furthermore, there were high correlations between the frequency of disadvantageous decisions in the Game of Dice Task and correct responses in the mWCST $(\rho=-.76, p<.001)$, and frequency of errors in the mWCST $(\rho=$ $.71, p<.001)$, but not the number of perseverations in the mWCST $(\rho=.29, p=.182)$. In addition, the frequency of disadvantageous decisions in the Game of Dice Task was negatively correlated with the copying of the $\operatorname{ROF}(\rho=-.42, p=$ .011). Further neuropsychological functions were not correlated with the frequency of disadvantageous decisions (see Table 3). To control for possible floor effects in anterograde memory scores (Memo delayed recall and ROF delayed recall) leading to nonsig- 


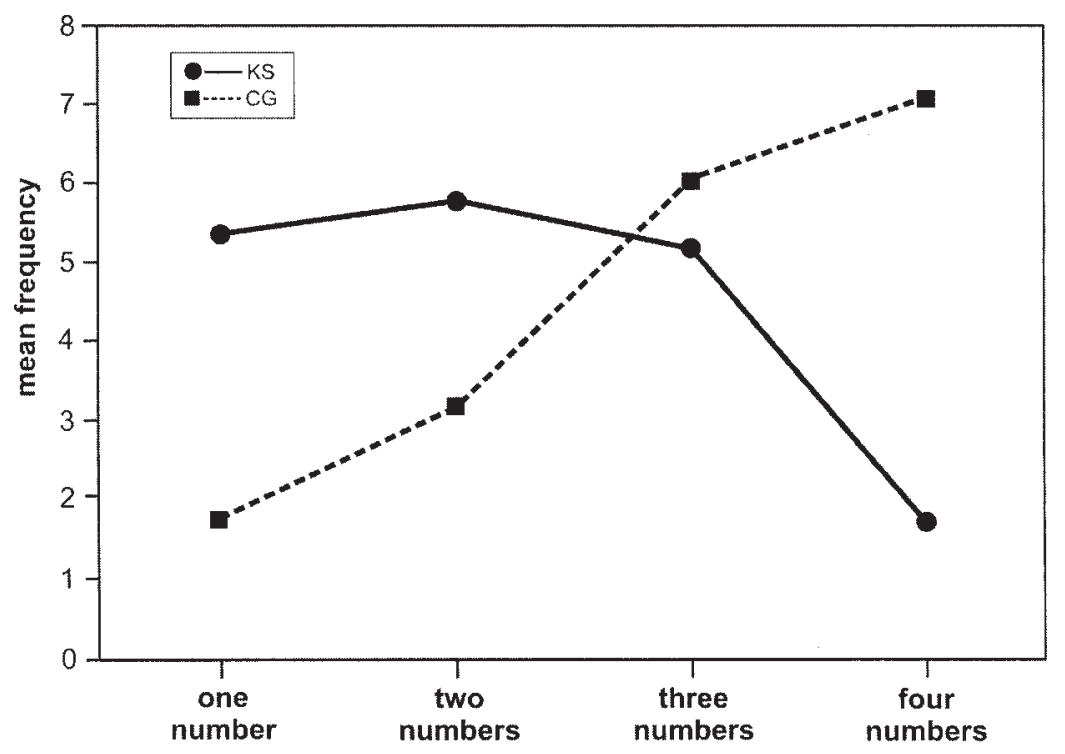

Figure 3. Mean frequency of each single alternative in the Game of Dice Task of the Korsakoff syndrome (KS) group and the control group (CG).

Table 3

Correlations in the Korsakoff Group Between the Frequency of Disadvantageous Decisions in the Game of Dice Task and Neuropsychological Tests



Note. $\quad$ FAS $=$ letters of the lexical verbal fluency task.

${ }^{\mathrm{a}}$ German: Farb-Wort-Test. ${ }^{\mathrm{b}}$ German: Mehrfachwahl-Wortschatz-Intelligenztest. ${ }^{\mathrm{c}}$ German: Hamburg-Wechsler-Intelligenztest für Erwachsene. nificant correlations with the Game of Dice Task performance, we split the Korsakoff group into two subgroups for each anterograde memory test (low scores vs. lowest scores), each group comprising approximately $50 \%$ of patients (split-half method). In the Memo delayed recall, 17 patients recalled zero items (Group Memo 0), and 18 patients recalled one or more items (Group Memo 1). The two subgroups did not differ significantly in the frequency of disadvantageous choices in the Game of Dice Task (Memo 0: median $=9.5$, range $=2-18 ;$ Memo $1:$ median $=13.5$, range $=$ $1-18 ; U=117.5, p=.358$ ). In the delayed recall of the ROF, 18 patients reached 4 points or less (Group Rey 0), and 17 patients revealed 5 points or more (Group Rey 1). Again, the subgroups did not differ regarding Game of Dice Task performance (Rey 0: median $=10.5$, range $=1-18 ;$ Rey $1:$ median $=13.0$, range $=$ $2-18 ; U=132.5, p=.503)$. Though subgroup analyses revealed no significance group differences, medians show a slightly better performance in the Game of Dice Task by those who were most impaired in anterograde memory.

Furthermore, to exclude the possibility that the correlation between performance in the mWCST (correct responses) and Game of Dice Task performance was mediated by anterograde amnesia, we conducted a nonparametric partial correlation analysis for the correlation between mWCST correct and disadvantageous decisions in the Game of Dice Task, controlling for Memo delayed recall. This analysis still revealed a significant correlation between Game of Dice Task disadvantageous choices and mWCST correct responses $(\rho=-.835, p<.001)$. In addition, the same correlation, with delayed recall of the ROF controlled for, was still significant $(\rho=-.749, p<.001)$.

In the controls, intelligence, general knowledge, and verbal fluency were not correlated with Game of Dice Task performance (all $p \mathrm{~s}>.05$ ). Because of the small neuropsychological test battery administered to the control participants in the current study, the possible relation of decision making in the Game of Dice Task and neuropsychological functions in healthy participants cannot be 
analyzed comprehensively. Nevertheless, unpublished data (Brand, Kalbe, Fujiwara, Kessler, \& Markowitsch, 2004) of a separate normative study of the Game of Dice Task give evidence that in healthy participants $(n=150,70$ men, 80 women, age $=$ $18-79$ years), task performance is unrelated to any cognitive or mnestic functions measured by standard neuropsychological tests. In this norm group, correlations between Game of Dice Task performance and intelligence (Reasoning subtest from the Leistungsprüfsystem; Sturm, Willmes, \& Horn, 1993), anterograde memory (Word List Learning and Word List Delayed Recall subtests of the DemTect; Kessler, Calabrese, Kalbe, \& Berger, 2000), working memory (Digit Span reverse), verbal fluency (Supermarket Task), and interference susceptibility (Interference trial of the Word Color Interference Task) were not significant (all $p \mathrm{~s}>$ $.05)$.

\section{Discussion}

Our main result is that KS patients are impaired in decision making in a gambling situation with explicit and stable rules for gains and losses, whereas healthy participants show risk avoidance. This was demonstrated both by the frequency of disadvantageous choices and by the selection of each single alternative. Furthermore, our results suggest that in KS patients, the frequency of disadvantageous decisions is highly correlated with specific subcomponents of executive functions, primarily, categorization, monitoring, and using of feedback for current decisions without a tendency toward perseveration (as measured by the mWCST). In fact, none of the KS patients and none of the healthy controls persisted on the choice they made the first time, but all participants changed to other alternatives at least within one category (risky decisions or nonrisky decisions). Thus, the high frequency of disadvantageous decisions in the Game of Dice Task seen in KS patients is not explained by a tendency to perseverate.

Some studies investigating both a gambling task and the WCST reported a dissociation of task performance (e.g., Bechara et al., 2001; Cavedini, Riboldi, D’Annucci et al., 2002; Cavedini, Riboldi, Keller et al., 2002). In our study, we found a significant correlation between decision-making performance and categorization, set-shifting, and cognitive flexibility as measured by the mWCST. These data indicate a strong association between decision making in our task with explicit and stable rules and the mentioned subcomponents of executive functions, even if mWCST performance was on average unimpaired in the KS group. Game of Dice Task performance may be mediated by subcomponents of executive functions as follows:

1. Participants have to plan to receive as much money as possible (goal definition).

2. They have to categorize the different alternatives regarding the associated risk. Here, they must classify alternatives according to their related gains and losses as well as to the different winning probabilities.

3. Participants may develop and apply a strategy to maximize profit.

4. They have to decide an alternative and to evaluate whether this decision was successful.

5. Participants may recognize that previous (risky) decisions were not successful and therefore have to shift to other alternatives that are more goal oriented. Alternately, if a choice was successful they may continue their strategy.

6. Furthermore, participants have to monitor how many rounds are left to play until the end of the game and may change their strategy accordingly with regard to the number of remaining trials.

Thus, the Game of Dice Task probably involves similar components of executive functions as the mWCST. The important difference between both tasks lies in two aspects: (a) In the mWCST, participants have to self-generate the exact current sorting rule, which is variable and differs across task duration. In the Game of Dice Task, the rules are explicit and stable. (b) The mWCST does not offer gains or losses, as does the Game of Dice Task. Therefore, the goals of both tasks differ. In the mWCST, the goal is to make as many correct decisions as possible, whereas in the Game of Dice Task, participants' primary goal is to gain as much money as possible within a defined number of trials. Furthermore, the Game of Dice Task is more complex regarding self-generation of goal-oriented strategies and decision monitoring. In KS patients, Game of Dice Task performance was correlated with correct response in the mWCST and with the number of nonperseverative errors, but not with perseverative errors. This suggests that their deficits in the Game of Dice Task are related to disturbances in categorization and evaluation of decisions, as well as in monitoring goal-oriented behavior and strategy. However, deficits in setshifting (in the sense of shifting between response alternatives) might have less impact on their decision-making performance.

Further correlations were found concerning risky decisions in the Game of Dice Task and the copying of the ROF, which is traditionally seen as assessing visuoconstructive abilities and the use of strategies (Lezak, 1995). One could argue that this correlation may be due to the fact that both tasks present visual stimuli; thus the finding may be the result of some KS patients' difficulties in organization of complex visual material. However, copying the ROF also requires action planning, monitoring, and cognitive flexibility (Lezak, 1995), which can be seen, for instance, in the correlation between copying the ROF and performance in the mWCST observed in our KS sample $(r=.649, p<.001)$. Accordingly, we interpret the correlation between copying the ROF and Game of Dice Task as evidence for common executive processes. These include the organization of the presented complex visual material and the categorization of different features of the material presented. Besides these executive components, visual working memory may also be involved in both copying the ROF and the Game of Dice Task. In both tasks, the visual input has to be held "online" to direct attention to the features relevant for the next step of task performance (e.g., to draw the next line in the ROF or to make the next choice in the Game of Dice Task). Future studies may investigate directly the potential impact of visual working memory on Game of Dice Task performance using a complex visual working memory task.

Aside from the aforementioned tests, no correlations between the frequency of disadvantageous decisions in the Game of Dice Task and other neuropsychological tests were revealed. Contrary to our hypothesis that decision making in a gambling situation with clear and stable rules for success depends on interference susceptibility, there was no such correlation. A possible explanation for 
this result might be that the task used to assess interference susceptibility in this study (Word Color Interference Test) measures interference susceptibility under a speed condition, whereas in the Game of Dice Task no such speed component in the decision-making process is demanded. Potentially, in the Game of Dice Task the attention and inhibition capacity (in the sense of selective attention for relevant stimuli and inhibition of irrelevant stimuli), as outlined in the introduction, is not linked to reaction time and interference susceptibility under a speed condition. In addition, the word-color interference effect based on Stroop (1935) might be caused by competition between an automatic response (lexical access activating response) and the production of a color name, or among the different task sets (for a discussion of probable basic mechanisms of interference, see Monsell, Taylor, \& Murphy, 2001). In the Game of Dice Task such a competition between automatic stimulus-response association does not exist. Instead, the type of input and response is equal for all alternatives (nonverbal choice of a number or a combination of numbers). Nevertheless, one could argue that when throwing a single die, the choice of a single number might appear easier than the choice of an alternative consisting of a set of numbers. Possibly, to switch between a task set of one single number (one die) and a combination of numbers is more difficult for the Korsakoff patients than for healthy controls. Following this argumentation, an interference effect would have influenced the worse performance of Korsakoff patients without being reflected in a correlation between wordcolor interference and Game of Dice Task performance. Future studies should investigate the possible interference effect on Game of Dice Task performance beyond the classical word-color paradigm, including tasks that assess higher order interference between different task sets. In addition, future studies could change the order of alternative presentation in the Game of Dice Task (alternatives with one single number are within the first line of alternatives so far; see Figure 1) to test whether the alignment of choices has impact on the costs of switching between different alternatives (and consequently task performance). In contrast to the interference mechanisms underlying the effects in a word-color interference paradigm (see Monsell et al., 2001), the potential interference in the Game of Dice Task lies in the competition between information given for the alternatives. One can hypothesize that the information of potentially high gains associated with the disadvantageous alternatives interferes with the information about the high loss and low winning probability of these alternatives. Therefore, other kinds of complex and emotional interference tasks should be used in future studies to assess this kind of interference and its possible influence on Game of Dice Task performance.

Furthermore, working memory was also uncorrelated with Game of Dice Task performance. This result is in accordance with a study by Bechara, Damasio, Tranel, and Anderson (1998), who reported a dissociation of performance in the Iowa Gambling Task and a working memory task. We expected a correlation between working memory and performance in our gambling task because working memory might be necessary for comparing different options and strategies. In contrast, we found only a minor role, if any, of working memory for decision-making process, at least in $\mathrm{KS}$ patients. One reason for this result may lie in the task procedure and presentation. The rules for gains and losses as well as the current capital in the Game of Dice Task are shown to the participants during the entire time of task accomplishment, and there- fore, working memory capacity does not seem to be crucial. However, the question remains whether visual working memory may impact Game of Dice Task performance, as mentioned above.

In addition, in our KS sample, no correlations between the frequency of disadvantageous decisions in the Game of Dice Task and different memory scores were observed. Even when we split the Korsakoff group into two subgroups according to their memory scores, no significant differences between participants with low and lowest memory performance were revealed. If anything, there was a slightly better performance in the Game of Dice Task by those who were poorest in anterograde memory. This result is in contrast to the study of Stout et al. (2001), who found a covariance between performance in the Iowa Gambling Task and the Conceptualization and Memory subscales of the Mattis Dementia Rating Scale (Mattis, 1988). An explanation for the controversial results of our study and that of Stout et al. may be that in the Iowa Gambling Task, the preference for advantageous card decks has to be learned by using the feedback from previous trials. In contrast, the Game of Dice Task displays its rules for gains and losses explicitly to the participants. Thus, winning probabilities can be easily reasoned. Instead, the challenge lies in selecting choices that result in the best long-term outcome. For this procedure, subcomponents of executive functions (see above) may be more important than learning and memory. Nevertheless, it cannot be excluded from our data that in Korsakoff patients, who have by definition severe anterograde memory disturbances, these deficits generally influenced decision-making performance but were not detectable with the explicit anterograde memory tests used.

In summary, the results of our study suggest that in a risky decision-making situation with explicit and stable rules for reinforcement and punishment, strategic decisions are possible, and therefore, specific subcomponents of executive functions attain substantial impact. Even though we found executive functions playing a major role for decision-making deficits in KS patients, it is also possible that these patients are deficient in the use of previous feedback for current decisions. Regarding the high negative balance of money at the end of the game in KS patients, one can assume that negative feedback did not trigger any strategy change in these patients. Possibly, a positive result in the Game of Dice Task might be attainable in two ways. In case of completely intact executive functions, strategic decisions to increase money long term are possible. Otherwise, if executive functions are impaired, feedback processing may enable advantageous choices. To evaluate this hypothesis, further research is needed to compare performance in both the Iowa Gambling Task and the Game of Dice Task in one sample of patients with frontal lobe damage or dysfunction together with neuropsychological tests, primarily assessing different aspects of executive functions.

\section{References}

Adinoff, B., Devous, M. D. S., Cooper, D. B., Best, S. E., Chandler, P., Harris, T., et al. (2003). Resting regional cerebral blood flow and gambling task performance in cocaine-dependent subjects and healthy comparison subjects. American Journal of Psychiatry, 160, 1892-1894.

American Psychiatric Association. (1994). Diagnostic and statistical manual of mental disorders (4th ed.). Washington, DC: Author.

Bechara, A. (2003). Risky business: Emotion, decision-making, and addiction. Journal of Gambling Studies, 19, 23-51. 
Bechara, A., Damasio, A. R., Damasio, H., \& Anderson, S. W. (1994). Insensitivity to future consequences following damage to human prefrontal cortex. Cognition, 50, 7-15.

Bechara, A., \& Damasio, H. (2002). Decision-making and addiction (Pt. I): Impaired activation of somatic states in substance dependent individuals when pondering decision with negative future consequences. Neuropsychologia, 40, 1675-1689.

Bechara, A., Damasio, H., Tranel, D., \& Anderson, S. W. (1998). Dissociation of working memory from decision making within the human prefrontal cortex. Journal of Neuroscience, 18, 428-437.

Bechara, A., Dolan, S., Denburg, N., Hindes, A., Anderson, S. W., \& Nathan, P. E. (2001). Decision-making deficits, linked to dysfunctional ventromedial prefrontal cortex, revealed in alcohol and stimulant abusers. Neuropsychologia, 39, 376-389.

Bechara, A., Dolan, S., \& Hindes, A. (2002). Decision-making and addiction (Pt. II): Myopia for the future or hypersensitivity to reward? Neuropsychologia, 40, 1690-1705.

Bechara, A., \& Martin, E. M. (2004). Impaired decision making related to working memory deficits in individuals with substance addictions. $\mathrm{Neu}$ ropsychology, 18, 152-162.

Bechara, A., Tranel, D., \& Damasio, H. (2000). Characterization of decision-making deficit of patients with ventromedial prefrontal cortex lesions. Brain, 123, 2189-2202.

Becker, J. T., Furman, J. M., Panisset, M., \& Smith, C. (1990). Characteristics of the memory loss of a patient with Wernicke-Korsakoff's syndrome without alcoholism. Neuropsychologia, 28, 171-179.

Bolla, K. I., Eldreth, D. A., London, E. D., Kiehl, K. A., Mouratidis, M., Contoreggi, C., et al. (2003). Orbitofrontal cortex dysfunction in abstinent cocaine abusers performing a decision-making task. NeuroImage, 19, 1085-1094.

Brand, M., Fujiwara, E., Kalbe, E., Steingass, H.-P., Kessler, J., \& Markowitsch, H. J. (2003). Cognitive estimation and affective judgments in alcoholic Korsakoff patients. Journal of Clinical and Experimental Neuropsychology, 25, 324-334.

Brand, M., Kalbe, E., Fujiwara, E., Huber, M., \& Markowitsch, H. J. (2003). Cognitive estimation in patients with probable Alzheimer's disease and alcoholic Korsakoff patients. Neuropsychologia, 41, 575584.

Brand, M., Kalbe, E., Fujiwara, E., Kessler, J., \& Markowitsch, H. J. (2004). [Normative data on the Game of Dice Task, gathered 20012004]. Unpublished raw data.

Bryan, J., \& Luszcz, M. A. (2000). Measures of fluency as predictors of incidental memory among older adults. Psychology and Aging, 15, 483-489.

Cavedini, P., Riboldi, G., D’Annucci, A., Belotti, P., Cisima, M., \& Bellodi, L. (2002). Decision-making heterogeneity in obsessive-compulsive disorder: Ventromedial prefrontal cortex function predicts different treatment outcomes. Neuropsychologia, 40, 205-211.

Cavedini, P., Riboldi, G., Keller, R., D’Annucci, A., \& Bellodi, L. (2002). Frontal lobe dysfunction in pathological gambling patients. Society of Biological Psychiatry, 51, 334-341.

Cravioto, H., Korein, J., \& Silberman, J. (1961). Wernicke's encephalopathy. Archives of Neurology, 4, 54-63.

D'Esposito, M., \& Grossman, M. (1996). The physiological basis of executive function and working memory. The Neuroscientist, 2, 345352.

Folstein, M. F., Folstein, S. E., \& McHugh, P. R. (1975). "Mini-MentalState"-A practical method for grading the cognitive state of patients for the clinician. Journal of Psychiatric Research, 12, 189-198.

Hughes, C. P., Berg, L., Danziger, W. L., Coben, L. A., \& Martin, R. L. (1982). A new clinical scale for the staging of dementia. British Journal of Psychiatry, 140, 566-572.
Joyce, E. M., \& Robbins, T. W. (1991). Frontal lobe function in Korsakoff and non-Korsakoff alcoholics: Planning and spatial working memory. Neuropsychologia, 29, 709-723.

Kessler, J., Calabrese, P., Kalbe, E., \& Berger, F. (2000). DemTect: Ein neues Screening-Verfahren zur Unterstützung der Demenzdiagnostik [DemTect: A new screening instrument to support dementia diagnosis]. Psycho, 26, 343-347.

Kessler, J., Markowitsch, H. J., \& Denzler, P. (1990). Der Mini Mental Status Test [The Mini Mental Status Test]. Weinheim, Germany: BeltzTest-Verlag.

Kopelman, M. D. (1991). Frontal dysfunction and memory deficits in the alcoholic Korsakoff syndrome and Alzheimer-type dementia. Brain, 114, 117-137.

Krabbendam, L., Visser, P. J., Derix, M. M. A., Verhey, F., Hofman, P., Verhoeven, W., et al. (2000). Normal cognitive performance in patients with chronic alcoholism in contrast to patients with Korsakoff's syndrome. Journal of Neuropsychiatry and Clinical Neurosciences, 12, 44-50.

Kril, J. J., Halliday, G. M., Svoboda, M. D., \& Cartwright, H. (1997). The cerebral cortex is damaged in chronic alcoholics. Neuroscience, 79, 983-998.

Lehrl, S., Merz, J., Burkhard, G., \& Fischer, S. (1991). MehrfachwahlWortschatz-Intelligenztest [Mehrfachwahl-Wortschatz Intelligence Test]. Göttingen, Germany: Hogrefe.

Lezak, M. D. (1995). Neuropsychological assessment (3rd ed.). New York: Oxford University Press.

Lineweaver, T. T., Bondi, M. W., Thomas, R. G., \& Salmon, D. P. (1999). A normative study of Nelson's (1976) modified version of the Wisconsin Card Sorting Test in healthy older adults. The Clinical Neuropsychologist, 13, 328-347.

Lishman, W. A. (1990). Alcohol and the brain. British Journal of Psychiatry, 156, 635-644.

Mair, W. G. P., Warrington, E. K., \& Weiskrantz, L. (1979). Memory disorder in Korsakoff's psychosis. Brain, 102, 749-783.

Malamud, N., \& Skillicorn, S. A. (1956). Relationship between the Wernicke and Korsakoff syndrome. Archives of Neurology and Psychiatry, 76, 585-596.

Manes, F., Sahakian, B., Clark, L., Rogers, R., Antoun, N., Aitken, M., \& Robbins, T. (2002). Decision-making processes following damage to the prefrontal cortex. Brain, 125, 624-639.

Markowitsch, H. J., Kessler, J., Bast-Kessler, C., \& Riess, R. (1984). Different emotional tones significantly affect recognition performance in patients with Korsakoff psychosis. International Journal of Neuroscience, 25, 145-159.

Markowitsch, H. J., Kessler, J., \& Denzler, P. (1986). Recognition memory and psychophysiological responses to stimuli with neutral or emotional content: A study of Korsakoff patients and recently detoxified and longterm abstinent alcoholics. International Journal of Neuroscience, 29, 1-35.

Mattis, S. (1988). Dementia Rating Scale: Professional manual. Odessa, FL: Psychological Assessment Resources.

Mitchell, D. G. V., Colledge, E., Leonard, A., \& Blair, R. J. R. (2002). Risky decisions and response reversal: Is there evidence of orbitofrontal cortex dysfunction in psychopathic individuals? Neuropsychologia, 40, 2013-2022.

Miyake, A., Friedman, N. P., Emerson, M. J., Witzki, A. H., Howerter, A., \& Wager, T. D. (2000). The unity and diversity of executive functions and their contributions to complex 'frontal lobe' tasks: A latent variable analysis. Cognitive Psychology, 41, 49-100.

Monsell, S., Taylor, T. J., \& Murphy, K. (2001). Naming the color of a word: Is it responses or task sets that compete? Memory \& Cognition, 29, 137-151. 
Moselhy, H. F., Georgiou, G., \& Kahn, A. (2001). Frontal lobe changes in alcoholism: A review of the literature. Alcohol \& Alcoholism, 36, 357368.

Nelson, H. E. (1976). A modified card sorting test sensitive to frontal lobe defects. Cortex, 12, 313-324.

Nelson, H. E. (1982). National Adult Reading Test. Windsor, England: NFER-Nelson.

Oslin, D., Atkinson, R. M., Smith, D. M., \& Hendrie, H. (1998). Alcohol related dementia: Proposed clinical criteria. International Journal of Geriatric Psychiatry, 13, 203-212.

Osterrieth, P. A. (1944). Le test de copie d'une figure complexe: Contribution à l'étude de la perception et de la mémoire [Test of copying a complex figure: Contribution to the study of perception and memory]. Archives de Psychologie, 30, 286-356.

Oswald, W. D., \& Fleischmann, U. M. (1997). Das Nürnberger-AltersInventar [The Nürnberger-Alters Inventory]. Göttingen, Germany: Hogrefe.

Rogers, R. D., Owen, A. M., Middleton, H. C., Williams, E. J., Pickard, J. D., Sahakian, B. J., \& Robbins, T. W. (1999). Choosing between small, likely rewards and large, unlikely rewards activates inferior and orbital prefrontal cortex. Journal of Neuroscience, 20, 9029-9038.

Sanfey, A. G., Hastie, R., Colvin, M. K., \& Grafman, J. (2003). Phineas gauged: Decision-making and the human prefrontal cortex. Neuropsychologia, 41, 1218-1229.

Schaaf, A., Kessler, J., Grond, M., \& Fink, G. (1992). Memo Test. Ein verbaler Gedächtnistest nach der Methode des selektiven Erinnerns [Memo Test. A verbal selective reminding test]. Weinheim, Germany: Beltz-Test-Verlag.
Shimomura, T., Mori, E., Hirono, N., Imamura, T., \& Yamashita, H. (1998). Development of Wernicke-Korsakoff syndrome after long intervals following gastrectomy. Archives of Neurology, 55, 1242-1245.

Smith, E. E., \& Jonides, J. (1999, March 12). Storage and executive processes in the frontal lobes. Science, 283, 1657-1661.

Spreen, O., \& Strauss, E. (1998). A compendium of neuropsychological tests (2nd ed.). New York: Oxford University Press.

Stout, J. C., Rodawalt, W. C., \& Siemers, E. R. (2001). Risky decision making in Huntington's disease. Journal of the International Neuropsychological Society, 7, 92-101.

Stroop, J. R. (1935). Studies of interference in serial verbal reactions. Journal of Experimental Psychology, 18, 643-662.

Sturm, W., Willmes, K., \& Horn, W. (1993). Leistungsprüfsystem für 50-90 jährige [Leistungsprüfsystem Intelligence Test for adults between 50 and 90 years]. Göttingen, Germany: Hogrefe.

Sullivan, E. V., \& Marsh, L. (2003). Hippocampal volume deficits in alcoholic Korsakoff's syndrome. Neurology, 61, 1716-1719.

Tewes, U. (1991). Hamburg-Wechsler-Intelligenztest für ErwachseneRevision [Hamburg-Wechsler Intelligence Test for Adults]. Göttingen: Hogrefe.

Victor, M., Adams, R. D., \& Collins, G. H. (1989). The WernickeKorsakoff syndrome (2nd ed.). Philadelphia: F. A. Davis.

World Health Organization. (1994). International statistical classification of diseases and health related problems, 10th version. Geneva, Switzerland: Author.

Received January 12, 2004

Revision received April 2, 2004

Accepted April 7, 2004 